\title{
Lung disease due to FLNA mutation improved after shunt closure for congenital heart disease
}

\author{
Satomi Mori ${ }^{1}$, Koji Tanoue $^{1}$, Hiroyuki Shimizu ${ }^{1}$, Hiroyuki Nagafuchi ${ }^{1}$, Ki Kim ${ }^{1}$, Hiroaki \\ Murakami $^{1}$, Kenji Kurosawa ${ }^{1}$, and Kiyoshi Matsui ${ }^{1}$ \\ ${ }^{1}$ Kanagawa Childrens Medical Center
}

November 26, 2020

\begin{abstract}
The FLNA gene encodes filamin A, an actin filament cross-linking protein that is ubiquitously expressed within the body. FLNA mutation causes periventricular nodular heterotopia (PVNH) and congenital heart disease. Interstitial lung disease (ILD) related to FLNA mutation has also been reported from 2011 and can be lethal. However, there are no reports of how to combine the treatment of heart disease with the conflicting treatment of lung disease. We herein report cases of two girls with FLNA mutation and both ILD and left-to-right shunts due to congenital heart disease. They presented with respiratory symptoms in early infancy and required management with long-term intubation and ventilation. However, their respiratory status improved subsequent to the closure of their left-to-right shunts even though they were small shunts with improvement in pulmonary hypertension. This suggests that early intervention with closure of cardiac shunts can prevent further deterioration of lung damage.
\end{abstract}

Title page

Lung disease due to FLNA mutation improved after shunt closure for congenital heart disease Satomi Mori MD ${ }^{1)}$, Koji Tanoue $\mathrm{MD}^{1)}$, Hiroyuki Shimizu MD ${ }^{2)}$, Hiroyuki Nagafuchi $\mathrm{MD}^{2)}$, Ki-Sung Kim $\mathrm{MD}, \mathrm{PhD}^{3)}$, Hiroaki Murakami MD, $\mathrm{PhD}^{4)}$, Kenji Kurosawa $\mathrm{MD}, \mathrm{PhD}^{4)}$, Kiyoshi Matsui $\mathrm{MD}^{1)}$.

1. Department of general medicine, Kanagawa Children's Medical Center, Yokohama, Japan

2. Department of critical care medicine, Kanagawa Children's Medical Center, Yokohama, Japan

3. Department of cardiology, Kanagawa Children's Medical Center, Yokohama, Japan

4. Division of medical genetics, Kanagawa Children's Medical Center, Yokohama, Japan

Corresponding author: Satomi Mori. Department of general medicine, Kanagawa Children's Medical Center, Yokohama, Japan

Funding Source: This work was supported by the Initiative on Rare and Undiagnosed Diseases (19ek0109301) and MGeND (Medical Genomics Japan Variant Database (19kk0205014) from the Japan Agency for Medical Research and Development and JSPS KAKENHI 20k08270 (K. Kurosawa).

Address: Mutsukawa 2- 138-4, Minami-ku, Yokohama, Kanagawa.

Postal code: 232-8555, Japan

Tel: +81-45-711-2351

Fax: +81-45-721-3324

E-mail:satomiforest@gmail.com 
KEYWORDS:FLNA, filamin A, interstitial lung disease, pulmonary hypertension

Running head: Shunt closure improves ILD due to FLNA mutation

Abstract

The FLNA gene encodes filamin A, an actin filament cross-linking protein that is ubiquitously expressed within the body. FLNA mutation causes periventricular nodular heterotopia (PVNH) and congenital heart disease. Interstitial lung disease (ILD) related to FLNA mutation has also been reported from 2011 and can be lethal. However, there are no reports of how to combine the treatment of heart disease with the conflicting treatment of lung disease. We herein report cases of two girls with FLNA mutation and both ILD and left-to-right shunts due to congenital heart disease. They presented with respiratory symptoms in early infancy and required management with long-term intubation and ventilation. However, their respiratory status improved subsequent to the closure of their left-to-right shunts even though they were small shunts with improvement in pulmonary hypertension. This suggests that early intervention with closure of cardiac shunts can prevent further deterioration of lung damage.

To the editor,

The FLNA gene encodes filamin A and is located at chromosome Xq28. Filamin A is an actin filament crosslinking protein and plays multiple roles in cell migration, blood vessel wall maintenance, signal transduction, and has been involved in tissue repair ${ }^{[1]}$.FLNA mutation is a well-known causative gene of periventricular nodular heterotopia (PVNH). Additionally, heterozygous loss-of-function mutations exhibit various human phenotypes, such as cardiovascular disease, joint hyperextension, and thrombocytopenia. Interstitial lung disease (ILD) related to FLNA mutation was first reported in $2011^{[2]}$. We present two cases of girls with FLNA mutation and both ILD and congenital heart disease (CHD). Both showed improved respiratory status following shunt closures.

\section{CASE 1:}

A girl weighing $2,520 \mathrm{~g}$ was born at 37 weeks' gestation. The patient developed difficulty in breathing, cough, and failure to thrive since the age of 3 months. Her oxygen saturation was $89 \%$ in air when she was admitted to our hospital at 4 months. Chest X-ray showed mildly increased diffuse pulmonary infiltrates. An echocardiogram revealed severe pulmonary hypertension and three left-to-right shunts: a small patent ductus arteriosus (PDA), atrial and ventricular septal defect. Chest computed tomography (CT) showed partial atelectasis in the bilateral dorsal region and hyperinflation in the upper lobe (Figure 1a, b). Cardiac catheterization revealed pulmonary arterial pressure of $56 / 28 \mathrm{mmHg}$ (mean, $37 \mathrm{mmHg}$ ) and the ratio of the pulmonary to systemic blood flow (Qp/Qs) was 1.1 in room air. Ambrisentan in addition to her regular dose of sildenafil was started. At the age of 5 months, the patient required intubation, ventilation for more than a month, and inhaled nitric oxide due to bronchiolitis caused by respiratory syncytial virus. Magnetic resonance imaging of the brain demonstrated bilateral PVNH. We performed exome analysis and identified a novel frameshift mutation c.4318delG:p.(Val1440Serfs*6) in FLNA. Sanger sequencing confirmed it was de novo.

The patient was re-intubated and ventilated at 7 months of age due to the sudden onset of desaturation without infection. She underwent PDA ligation with the use of an Amplatzer Vascular Plug device because of exacerbated pulmonary hypertension, pulmonary arterial pressure of 44/25 $\mathrm{mmHg}$ (mean, $34 \mathrm{mmHg}$ ), and Qp/Qs of 2.6. Her atrial and ventricular septal defects were closed to reduce the risk of increased afterload. The value of N-terminal pro-B-type natriuretic peptide (NT-proBNP) was $6,280 \mathrm{pg} / \mathrm{mL}$ when the patient was 5 months old, which decreased to $139 \mathrm{pg} / \mathrm{mL}$ following shunt closure, and the chest radiographs improved (Figure 1c-e). Her respiratory condition continued to improve, and she was discharged with high-flow nasal cannula oxygen therapy at the age of 1 year.

\section{CASE 2:}

A female neonate was born vaginally at 36 weeks' gestation with right full lip cleft. She was transported 
to our hospital due to cyanosis from birth. Although the echocardiogram showed aortic valve regurgitation, moderate pulmonary hypertension, and PDA; she was discharged without desaturation at 11 days old. She was admitted with poor feeding at 4 weeks old, and her oxygen saturation in room air fell to $83 \%$. Chest CT showed partial atelectasis in dorsal predominance and most of the right upper lobe. Lobular emphysematous changes with hyperinflation were observed in both lungs. The echocardiogram revealed a 2.5-mm PDA with a left-to-right shunt, and exacerbation of pulmonary hypertension. Sildenafil was administered for pulmonary hypertension. Her respiratory symptoms deteriorated, and she was intubated and ventilated. She was briefly extubated, but she required reintubation due to respiratory failure and ventilatory support. Cardiac catheterization revealed a pulmonary artery pressure of $46 / 27 \mathrm{mmHg}$ (mean, $36 \mathrm{mmHg}$ ). Pulmonary hypertension was suspected as the cause of the recurring breathing problems, so she underwent PDA ligation. She was successfully weaned off the ventilator and discharged at 8 months old on $0.5 \mathrm{~L} / \mathrm{min}$ of oxygen. After the left-to-right shunt had closed, her NT-proBNP value reduced from $8,973 \mathrm{pg} / \mathrm{mL}$ to $312 \mathrm{pg} / \mathrm{mL}$. Whole exome sequencing analysis was performed and detected a nonsense mutation c.7231C>T:p.(Arg2411*) in the FLNA gene.

We report two girls with FLNA mutation and both ILD and left-to-right shunts due to CHD who had difficulty in managing their respiration but showed improved respiratory status after shunt closure. Partnership with the cardiology, cardiovascular surgery, and intensive care departments led to successful treatment.

Respiratory disorders associated with FLNA mutation can be lethal. Some children have died of infection and respiratory failure, and others have received lung transplantation in Europe and the United States ${ }^{[2,3]}$.Our cases were primarily treated as hypoxia due to lung disease and the patients initially were administered with oxygen and pulmonary vasodilators. However, the respiratory status of both patients worsened, which could be attributed to not only ILD but also pulmonary over-circulation.

Pathological findings of the lung associated with FLNA mutations have been reported in the lung parenchyma with alveolar simplification and pulmonary artery curvature ${ }^{[3]}$. The increase in volume loading for poor vascular beds is seen in ILD with pulmonary hypertension. It is our opinion that as filamin A is capable of repairing tissues, its mutation could result in lung damage. Both patients showed improved breathing conditions after closing the small left-to-right shunts and were consequently discharged.

Pulmonary hypertension is a common comorbidity with ILD and is associated with a substantially increased mortality risk ${ }^{[4]}$. Our cases were complicated by pulmonary hypertension and CHD. High NT-proBNP levels are associated with a poor prognosis in ILD patients ${ }^{[5]}$. The NT-proBNP value of our cases decreased following shunt closure. The postoperative reduction in the cardiac volume load caused by the shunt suggested that pulmonary congestion improved, suggesting that NT-proBNP could also be used as a biomarker in this disease.

Despite the seemingly unrelated treatment between respiratory failure and heart failure, the outcome of our cases suggests that aggressive shunt closure intervention, even if the shunt is small, can improve the condition of lung damage and the prognosis of patients with lung disease associated with FLNA mutation.

Acknowledgements: This work was supported by the Initiative on Rare and Undiagnosed Diseases(19ek0109301) and MGeND (Medical Genomics Japan Variant Database(19kk020514) from the Japan Agency for Medical Research and Development and JSPS KAKENHI 20K08270(K.K.).

Conflict of interest: None

Reference

1. Robertson SP. Filamin A: phenotypic diversity. Curr Opin Genet Dev 2005;15:301-307.

2. Masurel-Paulet A, Haan E, Thompson EM, Goizet C, Thauvin-Robinet C, Tai A, Kennedy D, Smith G, Khong TY, Sole G, et al. Lung disease associated with periventricular nodular heterotopia and an FLNA mutation. Eur J Med Genet 2011; 54:25-28. 
3. Sasaki E, Byrne AT, Phelan E, Cox DW, Reardon W. A review of filamin A mutations and associated interstitial lung disease. Eur J Pediatr 2019;178:121-129.

4. Bromley S, Vizcaya D: Pulmonary hypertension in childhood interstitial lung disease; A systematic review of the literature. Pediatr Pulmonol 2017; 52:689-698.

5. Andersen CU, Mellemkjaer S, Nielsen-Kudsk JE, Bendstrup E, Simonsen U, Hilberg O: Diagnostic and prognostic role of biomarkers for pulmonary hypertension in interstitial lung disease. Respir Med 2012; 106:1749-1755.

Image legend: Axial chest computed tomography images of Case 1 of a) the upper b) and lower at four months of age show partial atelectasis in the bilateral dorsal region and hyperinflation in the upper lobe. Serial chest radiographs at c) four months, d) eight months, and e) one year of age reveal improvement in hyperinflation and atelectasis.
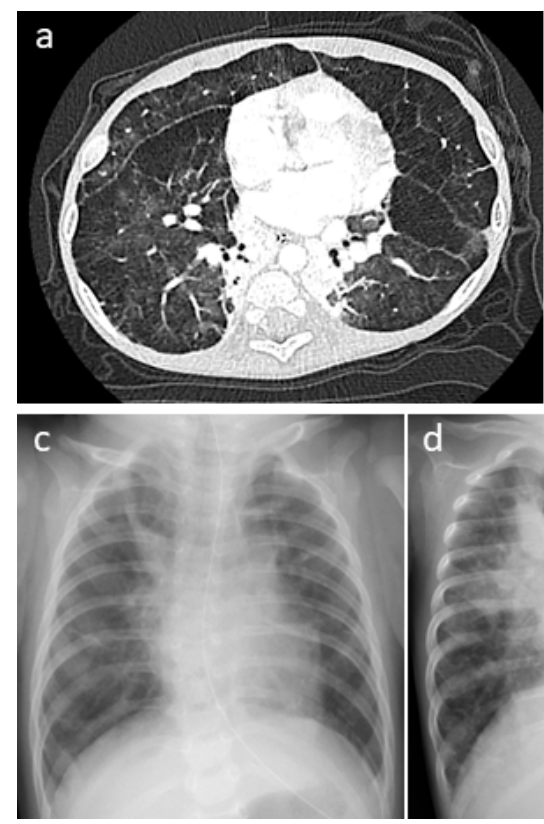
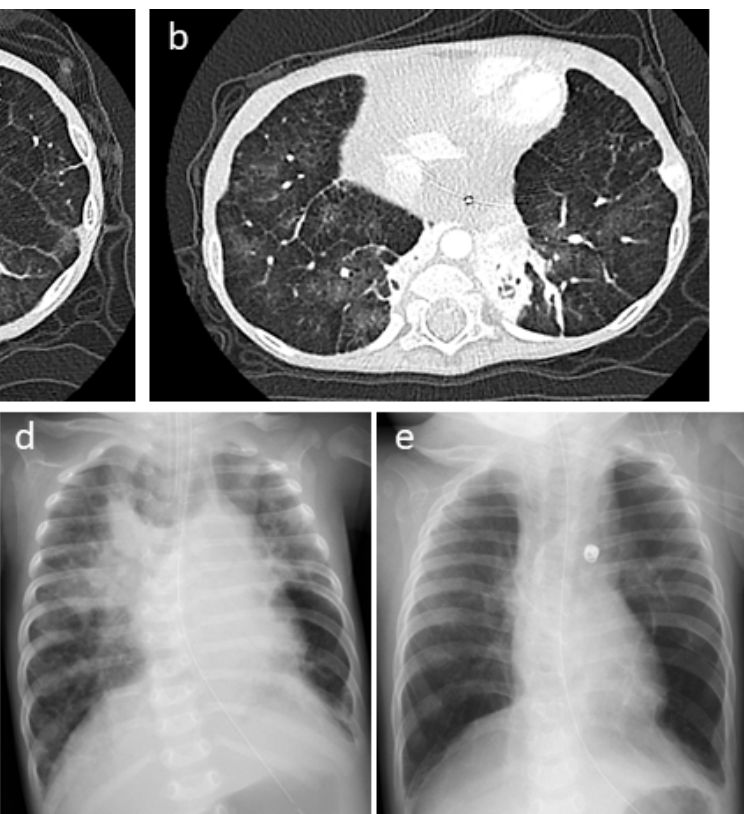